Louisiana State University

LSU Digital Commons

Faculty Publications

Department of Physics \& Astronomy

$1-1-2017$

\title{
Cosmology with Gravitational Waves in des and LSST
}

\author{
Kenneth Herner \\ Fermi National Accelerator Laboratory \\ Marcelle Soares-Santos \\ Fermi National Accelerator Laboratory \\ James Annis \\ Fermi National Accelerator Laboratory \\ G. González \\ R. Hynes
}

Follow this and additional works at: https://digitalcommons.Isu.edu/physics_astronomy_pubs

\section{Recommended Citation}

Herner, K., Soares-Santos, M., Annis, J., González, G., \& Hynes, R. (2017). Cosmology with Gravitational Waves in des and LSST. Proceedings of the International Astronomical Union, 13 (S338), 65-71.

https://doi.org/10.1017/S1743921318003794

This Conference Proceeding is brought to you for free and open access by the Department of Physics \& Astronomy at LSU Digital Commons. It has been accepted for inclusion in Faculty Publications by an authorized administrator of LSU Digital Commons. For more information, please contact ir@lsu.edu. 
$\begin{array}{ll}\text { Proceedings IAU Symposium No. 338, } 2017 & \text { (C) } 2017 \text { International Astronomical Union }\end{array}$

G. Gonzalez, R. Hynes, eds. DOI: $00.0000 / \mathrm{X} 00000000000000 \mathrm{X}$

\title{
Cosmology with Gravitational Waves in DES and LSST
}

\author{
Kenneth Herner $^{1}$, Marcelle Soares-Santos ${ }^{2,1}$, James Annis ${ }^{1}$ \\ on behalf of the Dark Energy Survey Gravitational Wave program \\ and the DECam Community Group Collaboration \\ ${ }^{1}$ Fermi National Accelerator Laboratory, Batavia, IL 60510, USA \\ email: kherner@fnal.gov \\ 2 Dept. of Physics, Brandeis University, Waltham, MA 02453, USA
}

\begin{abstract}
Motivated by the prospect of the wealth of data arising from the inauguration of the era of gravitational wave detection by ground-based interferometers the DES collaboration, in partnership with members of the LIGO collaboration and members of the astronomical community at large, have established a research program to search for their optical counterparts and to explore their use as cosmological probes. In this talk we present the status of our program and discuss prospects for establishing this new probe as part of the portfolio of the Dark Energy research program in the future, in particular for the next generation survey, LSST.
\end{abstract}

Keywords. (cosmology:) cosmological parameters, gravitational waves, stars: neutron, image processing

\section{Introduction}

One of the numerous breakthroughs that come from the dawn of gravitational wave (GW) astronomy is the ability to measure cosmological parameters. Perhaps the most natural parameter to measure is the Hubble Constant $H_{0}$, the expansion rate of the local universe. In turn the value of $H_{0}$ has consequences for other cosmological parameters such as the dark energy equation of state.

Merging compact binaries such as binary black hole (BBH) mergers, binary neutron star (BNS) mergers, and neutron star-black hole (NSBH) mergers can be used as socalled "standard sirens", to measure $H_{0}$ (Schutz 1986, Holz and Hughes 2005). Compact binaries can be broadly classified into two categories, "bright" and "dark" standard sirens, depending on whether they are expected to have an electromagnetic signature.

\subsection{Bright standard siren cosmology}

BNS and NS-BH events are known as "bright" standard sirens since there is an electromagnetic counterpart to the GW signal. Figure 1 illustrates how one can combine information from the GW waveform and from observations of the EM counterpart to measure the Hubble constant (Nissanke 2013). By identifying this counterpart and its host galaxy, if applicable, one can determine a redshift, and thus a recessional velocity as $v=c z$ at low redshift. After correcting for the peculiar velocity of the host relative to the background Hubble flow, one is left with a Hubble velocity $v_{H}$. This in turn leads to a determination of the Hubble constant via the relation $v_{H}=H_{0} d$, where $d$ is the distance to the source as measured from the GW signal.

The LIGO/Virgo Collaboration (LVC) has established partnerships with several collaborations around the world that perform observations across the EM spectrum to search for counterparts to GW event. Shortly after their initial analysis of a GW candidate, the

This document was prepared by DES and DECAM collaborations using the resources of the Fermi National Accelerator Laboratory (Fermilab), a U.S. Department of Energy, Office of Science, HEP User Facility. Fermilab is managed by Fermi Research Alliance, LLC (FRA), acting under Contract No. DE-AC02-07CH11359. 


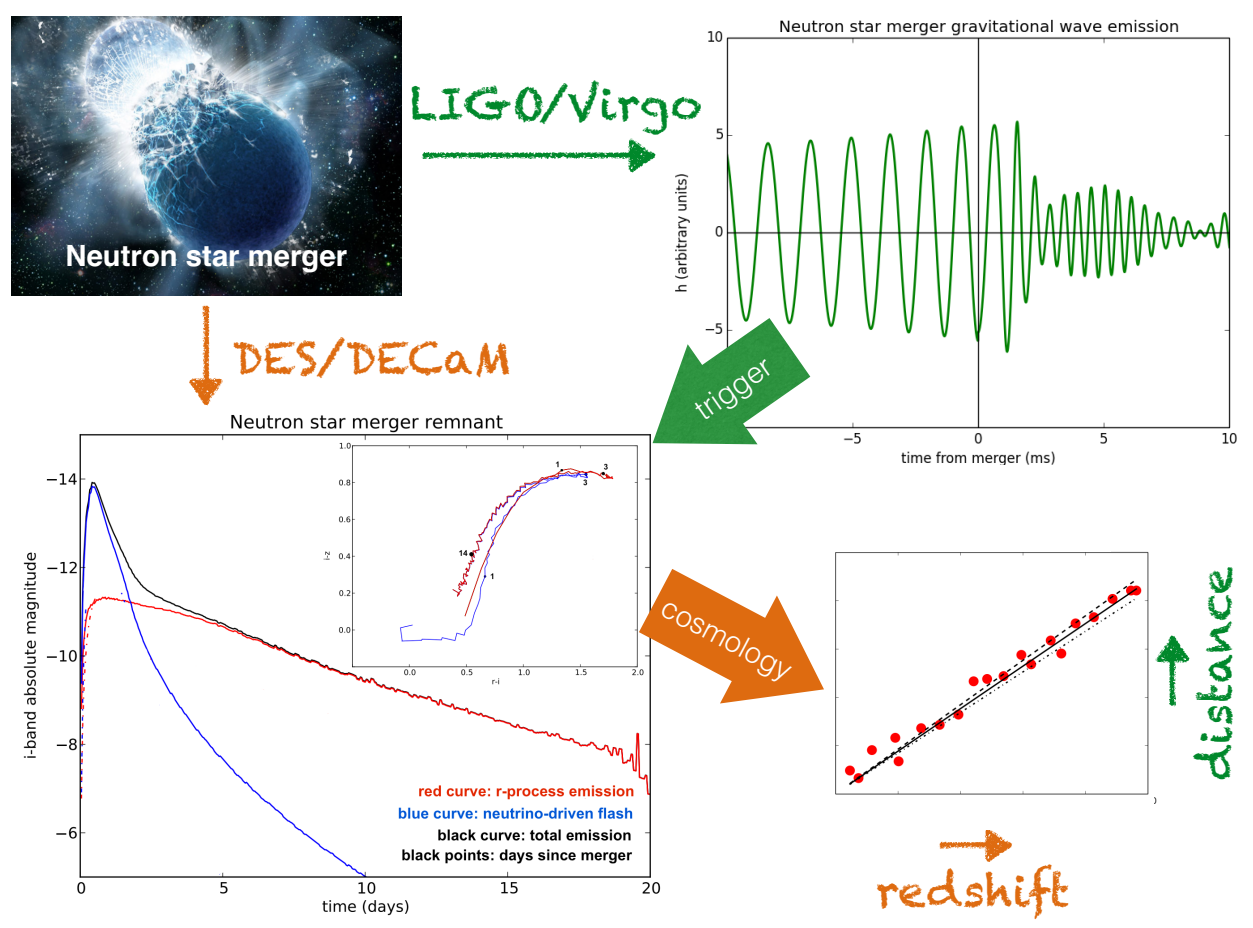

Figure 1. Diagram of the process to measure $H_{0}$ with bright standard sirens (BNS and NS-BH events). One can combine absolute distance measurement from the GW waveform and the redshift of the optical counterpart (or its host galaxy) and solve for $H_{0}$ using the relation $v_{H}=H_{0} d$, with $v_{H}=c z$ at low redshift.

LVC shares information with these partners, including estimated distance to the source, most likely type (e.g. BBH, BNS), and a map showing the probability of the source to be located at each point on the sky. The partner collaborations use this information to plan their follow-up campaigns.

\subsection{Dark standard siren cosmology}

$\mathrm{BBH}$ events can also form the basis of a $H_{0}$ measurement. In this case one can create a spatial map of BBH events using the localization and distance from LIGO. One can also make a map of galaxies and their redshift, as shown in Figure 2. By assuming a value for $H_{0}$, one can calculate a cross-correlation between the two maps. Varying the assumed value of $H_{0}$, one can build a cross-correlation distribution with the true value of $H_{0}$ being where the distribution is at its maximum.

\section{Overview of DESGW program}

One of the LVC EM followup partner groups is the Dark Energy Survey Gravitational Wave group (DESGW). The DESGW group consists of members of the Dark Energy Survey (DES) and other members of the astronomical community. DES built and is one of the primary users of DECam (Flaugher et al. 2015), at the $4 \mathrm{~m}$ Blanco Telescope at the Cerro Tololo InterAmerican Observatory in Chile. DECam is well-suited to the EM followup task due to its red-sensitive CCDs and its large aperture $\left(\approx 3 \mathrm{deg}^{2} \mathrm{FOV}\right)$. Once a trigger is received, our team uses a dedicated strategy code to decide whether or 


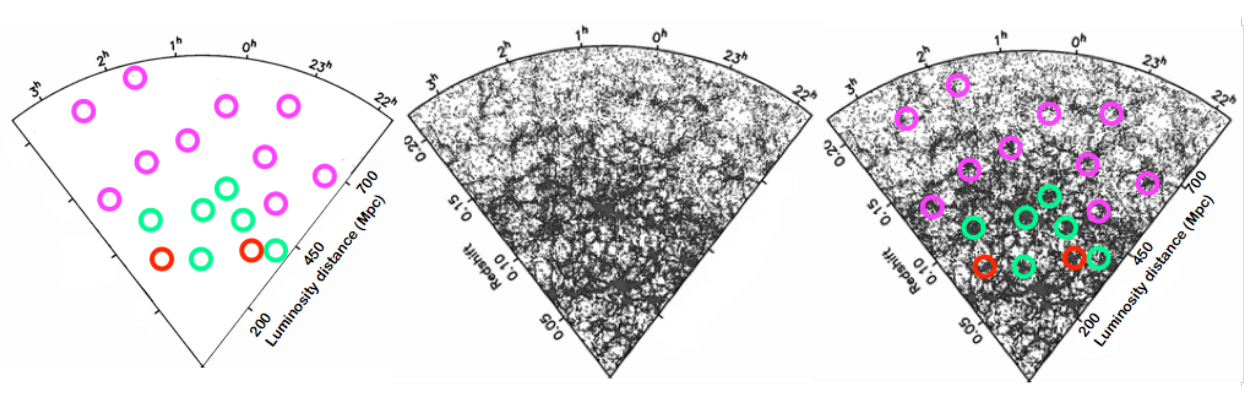

Figure 2. Measuring $H_{0}$ with dark standard sirens (BBH events). By comparing a map of BBH events (left panel; distances from LIGO measurements) with a map of galaxies (center panel; redshifts from catalogs), one can determine the value of $H_{0}$ by varying it and finding the value that maximizes the cross-correlation.

not to observe, how much telescope time to invest, and what sequence of observations to execute. If the decision is to observe, we interrupt DES observations to perform the search. Figure 3 illustrates the entire process from LIGO trigger, sent over the Gammaray Coordinates Network (GCN), to observations and candidate reporting, also via GCN circulars. The initial process is the same for BBH, BNS, and NS-BH events.

\section{Search program for bright gravitational wave sources}

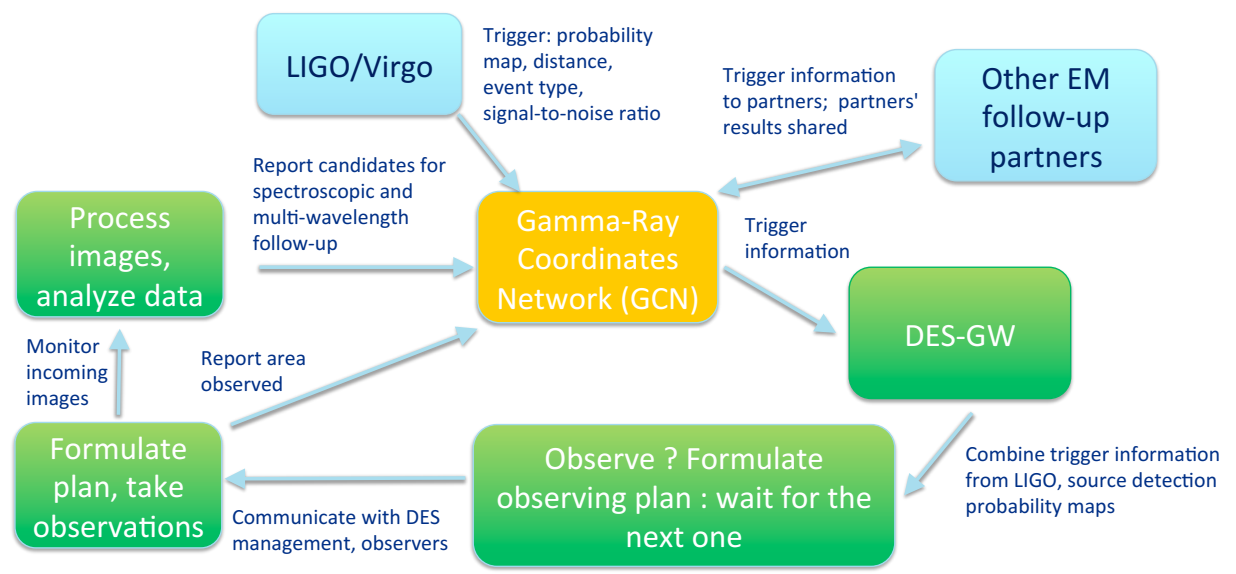

Figure 3. Overview of DESGW follow-up program. Communication between LIGO and EM partners is via GCN circulars. DESGW aims to complete image processing and candidate identification within 24 hours of data-taking.

See Herner et al. (2017) for further details of the observing plan construction and source models. In particular, we note that due in part to DECam's relatively wide field of view, we do not follow a strategy of targeting only galaxies consistent with the LIGO distance estimate. Instead we follow a "blind" search strategy, considering only the probability of detection based on expected source brightness and observing conditions.

To identify counterpart candidates we use the diffimg pipeline, described in Kessler et al. (2015). The pipeline takes search images and subtracts one or more "template" images taken of the same region of sky. Typically these images are earlier in time than the search images, but it is possible to use images taken after the search image (long enough for any expected objects to have faded below detection threshold) as templates if needed. We use all available DES images passing data quality requirements and $\geqslant 30 \mathrm{~s}$ in 
length, as well as publicly available DECam exposures of at least 30s taken on non-DES time.

Since the light from BNS events fades on the timescale of days, it is critical to rapidly complete image processing and candidate identification so that we can report any candidates in a GCN circular to alert other groups that may perform detailed spectroscopic followup. In particular we aim to complete analysis of each night's images in no more than 24 hours, with less than 12 hours being preferred. We have access to considerable grid computing resources to make such timescales possible, and we have succeeded in hitting our targets in $\mathrm{O} 2$.

\section{Results from LIGO trigger followup campaigns}

\subsection{Followup of BBH events}

As of the end of August 2017, DESGW performed four BBH optical counterpart searches, two in $\mathrm{O} 1$ and two in O2. Figure 4, upper left panel, shows the sky map for the GW150914 (Abbott et al. 2016a) followup campaign. The main counterpart search, described in Soares-Santos et al. (2016), covered approximately $40 \mathrm{deg}^{2}$ with 90 s exposures in $i$ and $z$. We are able to reach a depth of $i=22.1$ and $z=21.2$ in the subtracted images, for a source detection completeness of $>80 \%$. That translates into depth of 200 Mpc for a source with absolute magnitude, $M=-14$, similar to what one might expect for a kilonova. The merger emission model of Figure 1 assumes a search depth of 30Mpc. Note that the LIGO sensitivity in $2015-2016$ was $40-80 \mathrm{Mpc}$ for binary neutron stars.

Before it was known that GW150914 was a BBH merger, we considered that respected the spatial localization maps intersection with the Large Magellanic Cloud (LMC) and developed a hypothesis that the event could be a dark core collapse in the LMC. We imaged $34 \mathrm{deg}^{2}$ of the LMC region (Figure 4, upper right panel) with 5s exposures and searched for disappearance of previously cataloged red giant stars, the most likely to suffer a core collapse. All were accounted for. The LMC analysis Annis et al. (2016) allowed us to develop a template for future searches in case LIGO detects a nearby burst event and complements the main search. Our two analyses formed the most comprehensive optical search for signatures of GW150914 in terms of volume searched and methodology.

For the second LIGO event (GW151226; Abbott et al. 2016b), we performed only the main analysis (Figure 4, lower left panel) and covered a much smaller area because the region of interest was primarily in the north (Cowperthwaite et al. (2016)). That region also lacked pre-existing templates so we adapted our program to enable difference imaging with templates taken weeks after the trigger, when sources are expected to have faded. The analysis initially found four candidates; all were rejected as background upon further inspection. The longer timescale due to lack of full template coverage further motivates the pursuit of all sky-coverage with DECam. For this event a large fraction of the image processing was performed on local campus resources at Harvard and compared with the grid runs.

During the second LIGO observing season, O2, DESGW followed up GW170104 (LIGO Scientific Collaboration and Virgo Collaboration 2017a) and GW170814 (LIGO Scientific Collaboration and Virgo Collaboration 2017b). The follow-up search areas covered $13.6 \%$ and $84 \%$ of the LIGO $90 \%$ probability regions, respectively. We are analyzing both events to search for any possible electromagnetic counterparts. We are also performing a combined analysis of all BBH events we have studied to date; the chief goal is to set upper limits on optical emission from BBH events. 

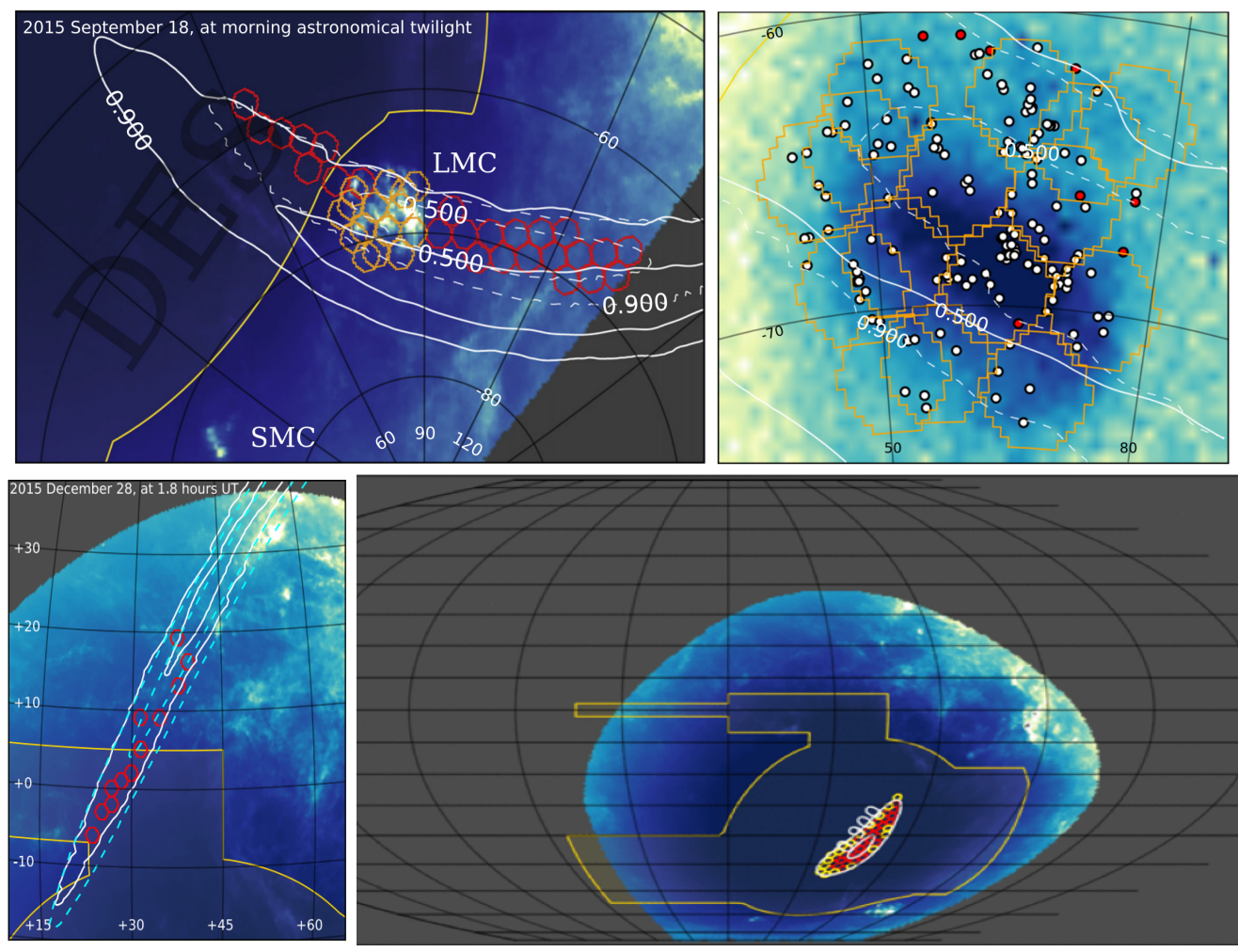

Figure 4. Sky maps for main followup of GW150914 (upper left), the disappearing star search in the LMC after GW150914 (upper right), the followup of GW151226 (lower left) and GW170814 (lower right). The white contours represent the $50 \%$ and $90 \%$ probability regions of the original (dashed) and updated (solid) LIGO probability maps. The yellow lines represent the fiducial DES observing region. The hexagons represent the locations of the telescope pointings during the search. In the upper left and both lower plots, the background colors indicate the DES limiting magnitude for observation, and in the upper right plot the background colors indicate stellar density.

\subsection{Followup of BNS events}

The first GW signal from a BNS event came on 17 August 2017 in the form of GW170817 (LIGO Scientific Collaboration and Virgo Collaboration 2017c), and triggered a massive EM follow-up campaign around the world Abbott et al. 2017. DESGW was one of several groups to independently identify the optical counterpart, and was able to exclude all other potential candidates within a large fraction $(>80 \%)$ of the LIGO probability region (Soares-Santos et al. 2017. We also performed detailed follow-up observations for nearly two week after the merger, and compare the data to several possible kilonova models in Cowperthwaite et al. (2017).

Our blind search strategy also enables the use of the redshift of the associated galaxy (NGC 4993) to calculate a value of $H_{0}$ in conjunction with the source distance estimate from the GW signal in LIGO Scientific Collaboration and Virgo Collaboration (2017d). The calculated value is $70.0_{-8.0}^{+12.0} \mathrm{~km} / \mathrm{s} / \mathrm{Mpc}$, consistent with results from supernovae (Reiss et al. 2016) and the CMB (Planck 2015), while also being independent of the cosmic distance ladder. 


\section{Future directions}

The third LIGO observing run is scheduled to begin in the summer of 2018. In the interim the DESGW group is working on a number of enhancements to its own program. These include gathering template images in regions where there is no existing DECam coverage, scaling up the overall data transfer rates, and building up a catalog of galaxy peculiar velocity measurements. This last item is particularly important for improving the accuracy of future $H_{0}$ measurements, since one must account for the peculiar velocity of a counterpart's host galaxy in order to obtain an accurate Hubble velocity.

The BNS detection rate will rise as LIGO's sensitivity increases. Starting from the present uncertainty of $\approx 15 \%$ on $H_{0}$ measured from GW170817, and extrapolating from Figure 2 of the DAWN II report ( LSC 2016) and assuming only improvements in uncertainty due to statistics, we can estimate the expected precision of GW-based $H_{0}$ measurements from KNe over time, shown here in Figure 5. We find that by 2019, we could expect the uncertainty to be at approximately $5 \%$, and by the start of the LSST era in 2022, a precision of $<2 \%$ should be possible with roughly 60 events.

Another possibility involves searches for untriggered kilonovae. The authors of Scolnic et al. (2017) have estimated how many KNe one might expect to identify without a LIGO trigger in several survey programs, including DES, WFIRST, and LSST. They find that relatively few kilonovae in completed and ongoing survey could be seen without a GW trigger, but several dozen could be seen with future surveys such as WFIRST and LSST. Indeed, WFIRST in particular should allow for kilonova detections out to $z \approx 0.8$, past the sensitivity of current GW detectors.

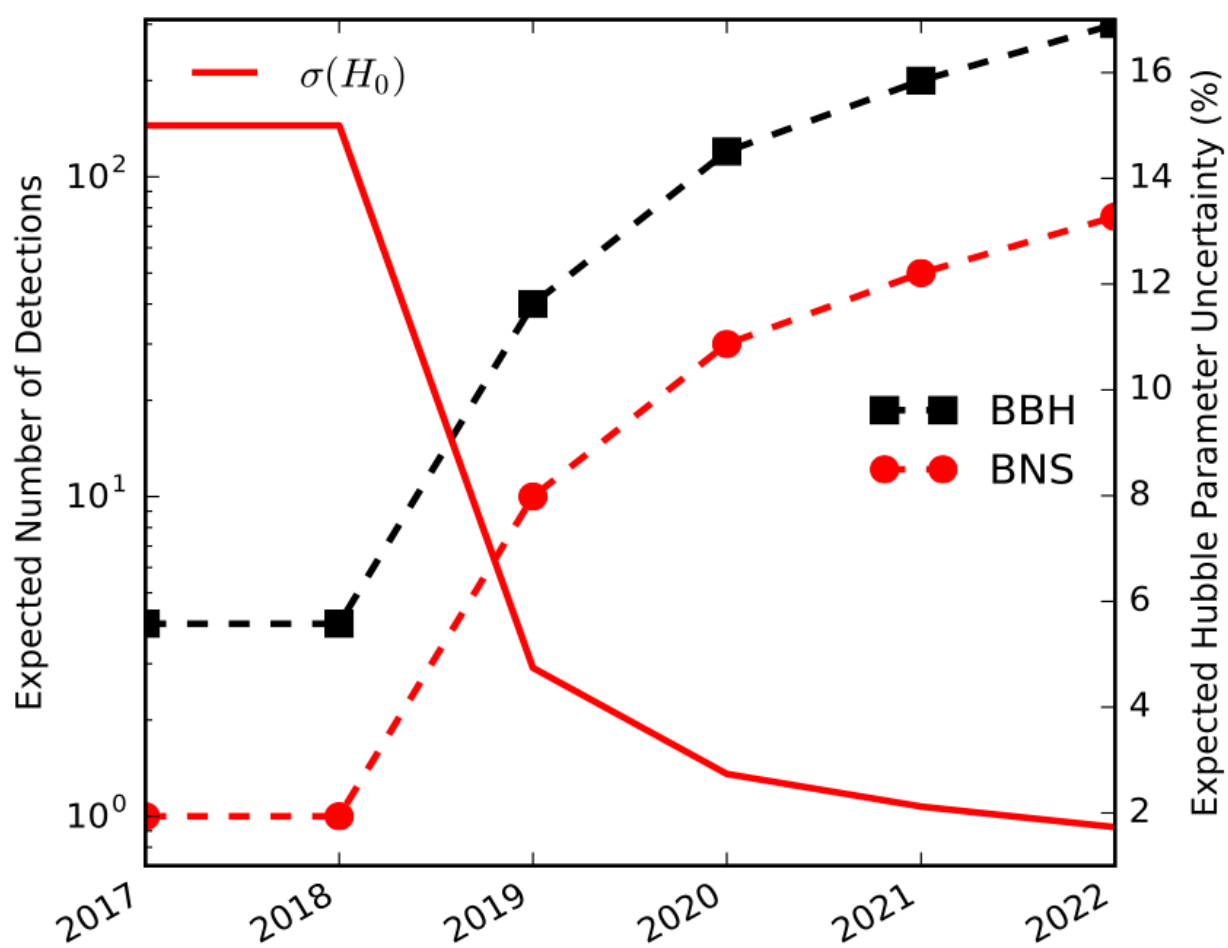

Figure 5. Expected number of BBH events (dark dotted line) and BNS events (light dotted line) detected by LIGO as a function of time. The solid line represents the expected uncertainty on $H_{0}$, assuming improvements in the statistical uncertainty due to the larger number of events. 


\section{Conclusions}

A source with the same absolute magnitude as GW170817, $M_{i}=-15.7$, would be bright enough for the DESGW program to detect it out to $425 \mathrm{Mpc}$. This detection has ushered in the era of multi-messenger astronomy, and enables independent measurements of cosmological parameters such as the Hubble constant. As GW detectors improve their sensitivity and additional join GW-EM observations occur, percent-level measurements of cosmological parameters should be possible at the dawn of the LSST era. Future survey projects, especially when combined with next-generation GW detectors, should provide an unparalleled window into the cosmos.

\section{Acknowledgments}

This research uses services or data provided by the NOAO Science Archive. NOAO is operated by the Association of Universities for Research in Astronomy (AURA), Inc. under a cooperative agreement with the National Science Foundation. This manuscript has been authored by Fermi Research Alliance, LLC under Contract No. DE-AC0207CH11359 with the U.S. Department of Energy, Office of Science, Office of High Energy Physics.

\section{References}

Abbott, B.P. et al., 2016, Phys. Rev. Lett., 116, 061102

Abbott, B.P. et al., 2016, Phys. Rev. Lett., 116, 241103

Abbott, B.P. et al., 2017, Phys. Rev. Lett., 118, 221101

Abbott, B.P. et al., 2017, Phys. Rev. Lett., 119, 141101

Abbott, B.P. et al., 2017, Phys. Rev. Lett., 119, 161101

LIGO Scientific Collaboration and Virgo Collaboration et al., 2017, Nature, 551, 85-88

Abbott, B.P. et al. 2017, ApJ (Letters), 848, L12

Annis, J. et al. 2016, ApJ (Letters), 823, L34

Cowperthwaite P. S., et al. 2016, ApJ (Letters), 826, L29

Cowperthwaite, P. S. et al. 2017, ApJ (Letters), 848, L17

Flaugher, B. et al. 2015, AJ, 150, 150

Herner, K. et al. 2017, J. Phys.: Conf. Ser., 898, 032050

Holz, D. E., Hughes, S. A. 2005, ApJ, 629, 15

Kessler R., Marriner J., Childress M. et al. 2015, AJ, 150, 172

LIGO Scientific Collaboration 2016, LIGO-P1600350

Nissanke, S. et al. 2013, arXiv:1307.2638

Planck Collaboration 2016, A\& A, 594, A13

Riess, A. G. et al. 2016, ApJ, 826, 56

Soares-Santos M., et al. 2016, ApJ (Letters), 823, L33

Soares-Santos, M. et al. 2017, ApJ (Letters), 848, L16

Schutz, B. F. 1986, Nature, 323, 310

Scolnic, D. et al. 2017, ApJ (Letters), in press. 